\title{
Measurement of Magnetic-Field Structures in a Laser-Wakefield Accelerator
}

\author{
M. C. Kaluza ${ }^{1,2,3}$, H.-P. Schlenvoigt ${ }^{1,4}$, S. P. D. Mangles ${ }^{3}$, A. G. R. Thomas ${ }^{3,5}$, \\ A. E. Dangor, ${ }^{3}$, H. Schwoerer, ${ }^{1,6}$, W. B. Mori, ${ }^{7,8}$, Z. Najmudin, ${ }^{3}$, and K. M. Krushelnick ${ }^{3,5}$ \\ ${ }^{1}$ Institut für Optik und Quantenelektronik, Friedrich-Schiller-Universität, 07743 Jena, Germany \\ ${ }^{2}$ Helmholtz-Institut Jena, Friedrich-Schiller-Universität, 07743 Jena, Germany \\ ${ }^{3}$ Department of Physics, Imperial College, London SW7 2AZ, United Kingdom \\ ${ }^{4}$ LULI, Ecole Polytechnique, 91128 Palaiseau cedex, France \\ ${ }^{5}$ Center for Ultrafast Optical Science (CUOS), University of Michigan, Ann Arbor, Michigan 48109 \\ ${ }^{6}$ Laser Research Institute, Stellenbosch University, Stellenbosch 7600, South Africa \\ ${ }^{7}$ Department of Physics and Astronomy, UCLA, Los Angeles, California 90095 and \\ ${ }^{8}$ Department of Electrical Engineering, UCLA, Los Angeles, California 90095
}

\begin{abstract}
Experimental measurements of magnetic fields generated in the cavity of a self-injecting laserwakefield accelerator are presented. Faraday rotation is used to determine the existence of multimegagauss fields, constrained to a transverse dimension comparable to the plasma wavelength $\sim \lambda_{p}$ and several $\lambda_{p}$ longitudinally. The fields are generated rapidly and move with the driving laser. In our experiment, the appearance of the magnetic fields is correlated to the production of relativistic electrons, indicating that they are inherently tied to the growth and wavebreaking of the nonlinear plasma wave. This evolution is confirmed by numerical simulations, showing that these measurements provide insight into the wakefield evolution with high spatial and temporal resolution.
\end{abstract}

PACS numbers: 52.38.Kd, 41.75.Jv, 29.30.Ep

The last few years have witnessed tremendous progress in the field of laser-driven electron acceleration. Electron beams with monoenergetic spectra can now be produced with high-power laser systems [1], potentially providing a source of brilliant secondary radiation [2]. Further improvements have now been made in terms of energy [3] and stability [4]. In these experiments, the highintensity laser pulse drives a plasma wave [5], which evolves into a 'bubble'-like structure with dimensions of order $\lambda_{p}=2 \pi c / \omega_{p}$ both transversely and longitudinally 1]. The 'bubble' compresses the laser pulse both spatially [6] and temporally [7] provided that it exceeds the power for relativistic self-focusing $P>P_{c r} \cong 17\left(n_{c r} / n_{e}\right)$ GW. This can lead to an increase in laser intensity eventually leading to wavebreaking and injection of electrons into the wave's electric field.

This high charge of accelerated electrons $(>100 \mathrm{pC})$, of ultrashort bunch duration $\left(\tau_{b}<\tau_{l} \simeq 50 \mathrm{fs}\right)$, thus constitutes an extremely high current $(>\mathrm{kA})$. Since the current is confined to a dimension much smaller than the plasma wave $(r \lesssim \mu \mathrm{m})$, it will generate a large associated azimuthal magnetic field, $B_{\varphi}=\mu_{0} I / 2 \pi r$. The field is not neutralized over distances comparable to the magnetic skin depth $\left(c / \omega_{p}\right)$ and over associated timescales $\left(1 / \omega_{p}\right)$, which are larger than those of the electron bunch. Hence these fields will serve to collimate the beam.

This is not the only magnetic field associated with the wakefield. In one dimension the coherent motion of electrons in the plasma wave do not produce a magnetic field, as the current due to the plasma oscillation $j_{c}=n e v$ exactly cancels the displacement current produced by the resulting charge separation $j_{d}=\epsilon_{0} \frac{\partial E}{\partial t}$. However in three dimension, electrons are pushed off-axis and return via the sheath of the 'bubble', so that there is a circulation of current in the wakefield which generates an azimuthal magnetic field [8]. In the 'bubble' regime the field can be calculated by considering the displacement current associated with the moving electron void $B_{\varphi} \simeq \frac{r_{\mathrm{b}}}{2} \frac{\partial E_{x}}{\partial(x-c t)}$ [9], where $r_{\mathrm{b}}$ is the blowout radius of the void and $E_{x}$ is the longitudinal electric field [10]. Hence the size of the field grows rapidly with plasma wave amplitude, and thus with increasing laser intensity. Thus measurements of these fields could provide important information which is difficult to obtain from highly non-linear wakefield accelerators [5], except from their radiation properties [11].

In this Letter, we present the first experimental evidence for the generation of multi-megagauss azimuthal magnetic fields in a laser wakefield. The evolution of the magnetic field indicates the rapid growth and subsequent breaking and decay of a large amplitude nonlinear plasma wave. Simulations confirm this evolution and show that the field is a combination of that due to the displacement current of the plasma wave as well as that associated with the current of relativistic electrons.

The experiments were performed with the 10-TW Ti:Sapphire JETI laser at the IOQ, Jena. Pulses of wavelength $\lambda=800 \mathrm{~nm}$, energy $E_{L}=800 \mathrm{~mJ}$, and duration $\tau=85$ fs were focused by an $f / 6$ off-axis parabola to intensity $I \simeq 3 \times 10^{18} \mathrm{Wcm}^{-2}$ onto the rising edge of an interferometrically characterized helium gas jet from a 1-mm diameter nozzle (Fig.11). Accelerated electrons were either detected by a fluorescent screen shielded by a $30 \mu \mathrm{m} \mathrm{Al-foil} \mathrm{to} \mathrm{block} \mathrm{laser} \mathrm{light}$ and a CCD-camera or alternatively by a high-resolution magnetic spectrometer. Quasi-monoenergetic features peaked around $50 \mathrm{MeV}$ were observed in the electron spectrum as in [12]. A synchronized probe pulse was gen- 


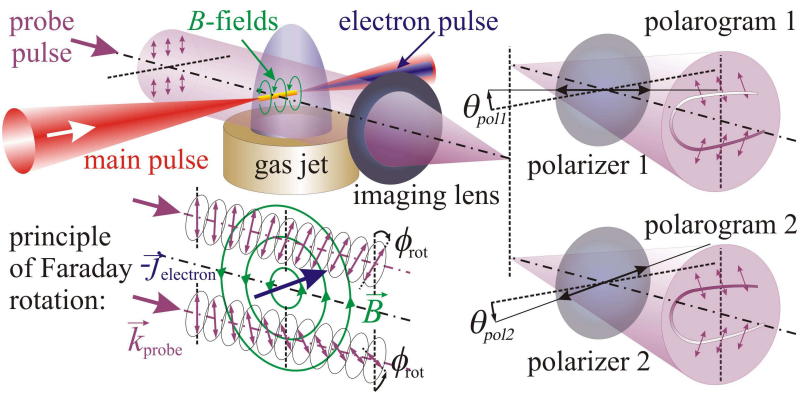

FIG. 1: Experimental set-up. An electron beam is generated by a self-injecting laser wakefield accelerator. A linearly polarized probe pulse traverses the interaction region and experiences Faraday rotation. The interaction region is imaged onto two CCD-cameras, each analysed with a polarizer.

erated from the transmission of the laser pulse through a 1:100 beam splitter. The probe was telescoped down and guided into the interaction chamber via a variable delay line [13]. Due to group-velocity dispersion when propagating through glass, the probe duration was slightly increased to $100 \mathrm{fs}$. The interaction region was backlit by the probe, which was then split by a non-polarizing beam splitter and imaged by a high-quality $f / 10$ lens with a magnification of 10 onto a pair of CCD-cameras, equipped with interference filters. The spatial resolution was $10 \mu \mathrm{m}$ in the transverse and $30 \mu \mathrm{m}$ in the laser direction, determined by the imaging system and due

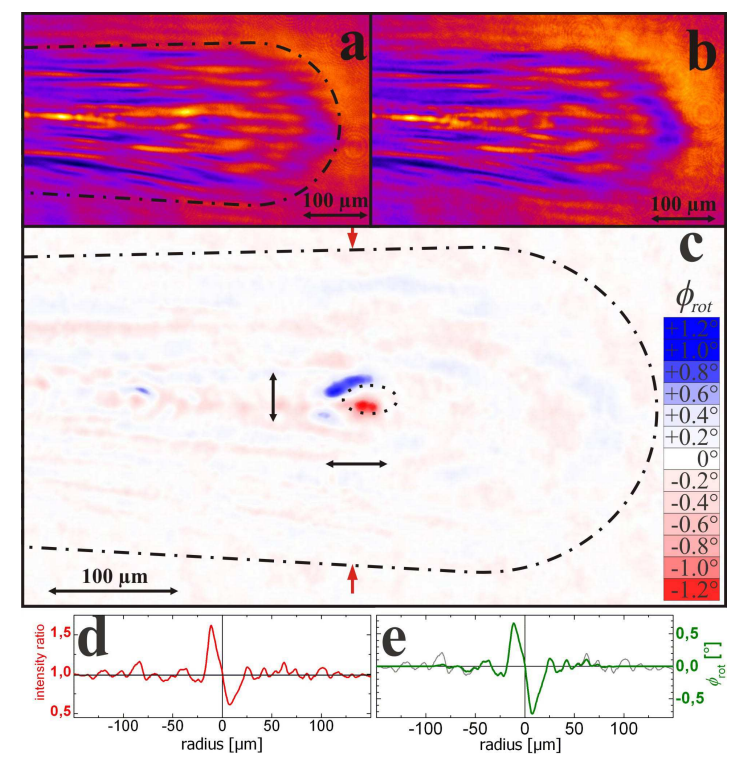

FIG. 2: Two polarograms in false colour for same laser shot with polarizer angles: a) $\theta_{\text {pol1 }}=+5.9^{\circ}$; b) $\theta_{\text {pol2 }}=-4.1^{\circ}$ ); c) distribution of $\phi_{\text {rot }}$ deduced from the intensity ratio of a) and $\mathrm{b})$. Most pronounced rotation occurs in a small region of (55 \pm 5$) \mu \mathrm{m} \times(35 \pm 5) \mu \mathrm{m}$ indicated by black arrows; d) line-out between the red arrows shown in c); e) associated $\phi_{\text {rot }}$. to the motion blur caused by the orthogonal motion of the main beam relative to the probe. In addition, polarizers (extinction ratios of $\left(1-\beta_{1}\right)=4 \times 10^{-3}$ and $\left.\left(1-\beta_{2}\right)=4 \times 10^{-4}\right)$ were positioned in front of each camera. A Nomarskii interferometer [14] was used to measure the electron density.

The $B_{\varphi}$-field distribution during the interaction was measured using Faraday rotation [15]. When $B_{\varphi}$ has components parallel to the propagation of the probe, the probe polarization is rotated by $\phi_{\text {rot }}$, (Fig.11). The accumulated rotation is $\phi_{\text {rot }}=\frac{e}{2 m_{\mathrm{e}} c n_{\text {cr }}} \int_{\ell} n_{\mathrm{e}}(r) \vec{B}_{\varphi}(r) \cdot \mathrm{d} \vec{s}$ taken along the path $\ell$ of each ray of the probe through the plasma. Here, $n_{\mathrm{cr}}=\varepsilon_{0} m_{\mathrm{e}} \omega_{\mathrm{pr}}^{2} / e^{2}$ the critical density for the probe light of frequency $\omega_{\text {pr }}$ and wavenumber $k_{\mathrm{pr}}=\omega_{\mathrm{pr}} / c$. To measure the distribution of $\phi_{\text {rot }}$, the two cameras were carefully aligned to take two images of the same interaction region. The two polarizers were detuned in opposite directions by $\theta_{\mathrm{pol}, 1}$ and $\theta_{\mathrm{pol}, 2}$ from the positions crossed with respect to the initial probe polarization. The intensity transmitted through polarizer $i$ (where $i=1,2$ ) can then be described by Malus' law,

$$
I_{\mathrm{pol}, i}=I_{0}\left[1-\beta_{i} \sin ^{2}\left[90^{\circ}+\theta_{\mathrm{pol}, i}+\phi_{\mathrm{rot}}\right]\right],
$$

where $I_{0}$ is the initial probe intensity. Taking two simultaneous polarograms eliminated shot-to-shot fluctuations of the probe-beam profile including plasma refraction effects, thus increasing the sensitivity of our measurements.

Fig. (2a) and b) show two such polarograms. The main pulse has entered the plasma from the left; intensity differences can be seen in the centre of the images. This is even more pronounced in the distribution of $\phi_{\text {rot }}$ (Fig.22) ), deduced from the ratio of the intensities of the two polarograms using eq. (11). The regions where the strongest rotation occurs are confined to the center of the image, being symmetric around the laser axis and having a longitudinal extent $(55 \pm 5) \mu \mathrm{m}$ and a transverse diameter of $(35 \pm 5) \mu \mathrm{m}$. The main pulse was situated slightly in front of this region, indicated by the dotted ellipse in Fig.2r). The position of the main pulse was determined by the appearance of ring-like interference in the images on separate shots where the probe intensity was reduced to a level comparable to the side-scattered light from the main pulse. As the interference can only be generated when the probe and scattered light overlap in space and time, the center of these rings marks the instantaneous position of the main pulse. A lineout of the intensity ratio through this region and of the associated Faraday-rotation angle are shown in Fig.2d) and e). The appearance of the intensity variations was correlated with the generation of an electron beam.

Two-dimensional (2D) simulations of the interaction were performed using the particle-in-cell code OSIRIS [16]. The plasma density profile was set to be gaussian $\left(1 / e^{2}\right.$ radius of $550 \mu \mathrm{m})$ with a peak density of $4 \times 10^{19} \mathrm{~cm}^{-3}=$ $0.023 n_{\mathrm{cr}}$ as in the experiment. A laser pulse of gaussian 
temporal profile with $\tau=70$ fs (FWHM), and vacuum focus of $6 \mu \mathrm{m}\left(1 / e^{2}\right.$ intensity radius) corresponding to $I=5.4 \times 10^{18} \mathrm{Wcm}^{-2}$ was set to focus $430 \mu \mathrm{m}$ before the peak density. A slightly higher initial intensity was chosen since 2D simulations tend to underestimate effects such as self-focusing which are important in the experiment. The simulations were performed in a box size of $800 \times 500 c / \omega_{0}$ with $4000 \times 1000$ grid cells with 2 macroparticles per cell. The box moved at the speed of light in the lab frame to allow higher resolution.

Fig. (3a) shows the electron density and the laser intensity from the simulation at the time when $B_{\varphi}$ is maximum. Due to the evolution of the laser, the plasma

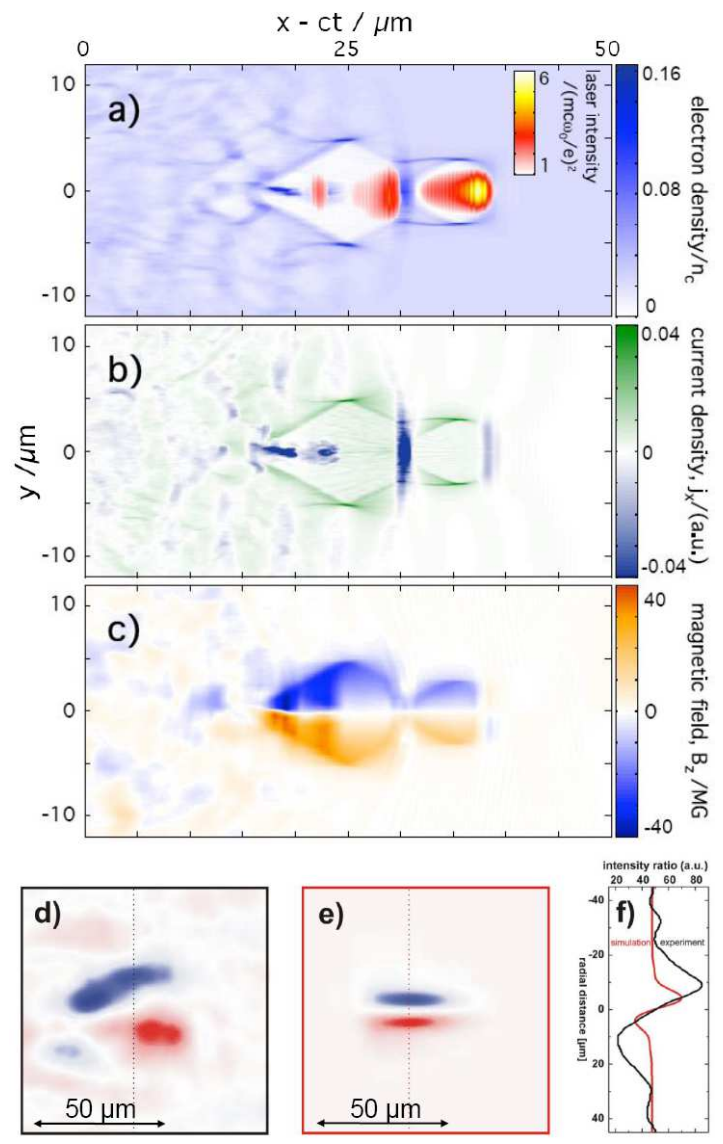

FIG. 3: Simulation results: a) electron density (blue) overlaid with laser intensity (red/yellow), b) longitudinal current density, c) $B_{\varphi}$ d) $92 \mu \mathrm{m} \times 92 \mu \mathrm{m}$ section of Fig.2 2 ), e) intensity modulation obtained from the numerical simulation including all effects affecting the image formation in the experiment, $\mathrm{f}$ ) vertical line-outs along the dotted lines in d) and e).

wake has evolved from an initially multi-period wave to a 'double-bubble' structure. Self-modulation of the pulse has occurred in both the longitudinal and transverse dimensions; the resulting pulselets contain sufficient energy to drive a highly non-linear wake structure [12, 17]. Both 'bubbles' have a strong $B_{\varphi}$-field and are of a similar size, Fig. [35). The first 'bubble' has a field of magnitude $B_{\varphi} \sim 15 \mathrm{MG}$, whereas for the second 'bubble', $B_{\varphi} \sim 35 \mathrm{MG}$. However, only the second 'bubble' has an on-axis current of trapped electrons generating a $B_{\varphi}$-field (Fig. 3b) indicating that there must be additional contributions to the field which is present in both 'bubbles'. This is evidently due to the displacement current. Both $B_{\varphi}$ contributions are orientated in the same way (left handed in direction of laser propagation). Even though the peak $B_{\varphi}$ lies within the 'bubble', where the density is low, in general there is not complete cavitation and the $B_{\varphi}$ spreads into the high-density walls of the 'bubble' leading to a measureable Faraday-rotation signal. Note, however, that the $B_{\varphi}$-fields are mainly confined to the 'bubble' structure due to return currents carried by background electrons flowing around both 'bubbles'.

For a quantitative comparison between experiment and simulation the numerical results shown in Figs. (3h)-c) were post-processed with a routine explicitly taking into account experimental effects influencing the image formation. Based on a ray-tracing routine accounting for refraction of the probe in the plasma, we further included the limited spatial resolution of the imaging system together with the limited temporal resolution due to the finite probe pulse duration of $100 \mathrm{fs}$ and the blurring caused by the perpendicular motion of the main pulse within the duration of the probe. These effects serve to increase the apparent size of the feature and also reducing the signal's amplitude. A comparison between the $B_{\varphi}$-field feature's spatial extent and a vertical lineout of the intensity distribution from the experiment and the post-processed simulation data are shown in Figs. (3d)-f). A good agreement is found both in size and amplitude. For a field of multi-megagauss strength to be generated by the accelerated electrons, implies a current of such electrons $>10 \mathrm{kA}$. The simulation shows that such large value of field and current are only possible because the pulse duration of the electrons is much shorter than the plasma wave duration (of order $\sim \mathrm{fs}$ ).

To separate the contribution to $B_{\varphi}$ by the wake displacement current from that from the electron-current, the individual quasi-particle momenta in the simulation was recorded. A particle was considered to be trapped if its forward momentum $p_{x}>7 \mathrm{MeV} / c$, corresponding to the velocity of the plasma 'bubble' for $n_{\mathrm{e}}>9 \times 10^{18} \mathrm{~cm}^{-3}$ and $\lambda_{0}=800 \mathrm{~nm}[9]$. From the current density of all trapped particles the associated azimuthal magnetic field is calculated. It increases rapidly at the same time as the total $B_{\varphi}$ confirming that for our experimental conditions the generation of $B_{\varphi}$ is strongly correlated with the trapped electron current. However, the accelerated electron alone only accounts for approximately half of the total $B_{\varphi}$-field (as shown by the circles in Fig. (4), confirming the importance of the $B_{\varphi}$-field due to the 'bubble' itself.

By controlling the delay between main and probe pulse, the temporal evolution of the magnetic fields 
was visualized (Fig.4a-f). Starting from the first image where no clear signature can be seen, the feature appears and reaches a maximum rapidly, but then becomes weaker again for later times. The distance between the 'bubble'-positions in two subsequent images which were delayed by $200 \mathrm{fs}$ stays approximately constant at $(60 \pm 5) \mu \mathrm{m}$ leading to a propagation velocity of the 'bubble' of $(3.00 \pm 0.25) \times 10^{8} \mathrm{~ms}^{-1}$, matching the laser's group velocity in the plasma.

In Fig. 4 $4 \mathrm{~g}$ ), the evolution of the peak Faraday-rotation angle, which is proportional to the $B_{\varphi}$ and which has been measured at different times in the plasma, is compared to the peak $B_{\varphi}$ from the simulation. After a rapid increase close to the peak of the density, both quantities show a slower decrease towards the end of the plasma. This rapid onset and the subsequent evolution both of

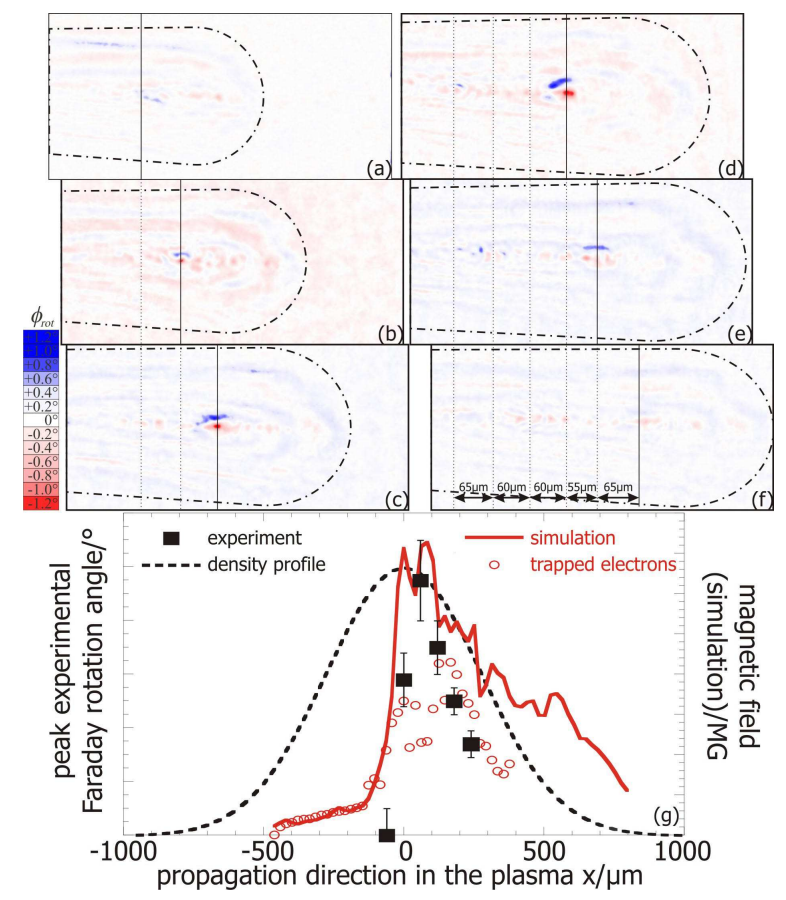

FIG. 4: a)-f) Time evolution of $B_{\varphi}$. Each image $(560 \mu \mathrm{m} \times$ $270 \mu \mathrm{m})$ shows distribution of $\phi_{\text {rot }}$. g) Peak $\phi_{\text {rot }}$ (squares) as function of position compared to corresponding $B_{\varphi}$ from simulation (red). Relative plasma density profile is indicated by dashed line. Circles denote $B_{\varphi}$-contribution from trapped electrons. Error determined by difference between peak value of $\phi_{\text {rot }}$ above and below laser axis.

the rotation and the $B_{\varphi}$-field imply a rapid steepening and breaking of the plasma wave and subsequent injection of background electrons into the wake. From these measurements, it is evident that the wave breaks only once, rather than a continual injection which is seen in simulations at higher intensity. The experimental measurements decay faster than $B_{\varphi}$ in the simulation. Hence it is apparent that in the experiment either the confinement of accelerated electrons and/or the plasma wave amplitude decays much faster than in the idealized conditions of the simulation. This is the first visualization of such non-ideal evolution of a plasma wave, and can help to explain the often large discrepancies between experiments and simulations.

In conclusion, we have measured the evolution of magnetic field structures during the interaction of a highintensity laser pulse with underdense plasma. This is closely related to the process of non-linear plasma wave formation and its breaking into a 'bubble'-like structure. The sudden onset of the $B_{\varphi}$-field is a clear signature of the non-linear steepening of the plasma wave and, for many experimental conditions, a signature of the subsequent injection of electrons into the wakefield. By reducing the plasma density below the threshold for electron self-injection our technique could also be used to detect the $B$-fields of the bubble alone. Our diagnostic gives experimentalists a powerful tool that can easily be implemented into existing set-ups to provide information of the acceleration process with high resolution. The results can directly be compared with numerical simulations which presently are the basis of our understanding of the acceleration processes in the plasma, eventually enabling researchers to identify crucial experimental parameters required for a stable interaction. This will help in optimizing laser-driven electron acceleration, bringing the prospect of table-top sources of brilliant and ultra-short XUV and x-rays closer to reality.

This work was supported by DFG (TR18) and BMBF (03ZIK052). We thank B. Beleites and F. Ronneberger for their excellent laser support. SPDM thanks the Royal Society and WBM thanks US DOE and NSF for funding.

[1] A. Pukhov and J. Meyer-ter-Vehn, Appl. Phys. B 74, 355 (2002); S. P. D. Mangles et al., Nature 431, 535 (2004); C. G. R. Geddes et al., ibid. 431, 538 (2004); J. Faure, et al., ibid. 431, 541 (2004).

[2] W. P. Leemans et al., Phys. Rev. Lett. 91, 074802 (2003); A. Rousse et al., ibid. 93, 135005 (2004); H.-P. Schlenvoigt et al., Nature Phys. 4, 130 (2008).

[3] W. P. Leemans et al., Nature Phys. 2, 696 (2006); S. Kneip et al., Phys. Rev. Lett. 103, 035002 (2009)

[4] J. Faure et al., Nature (London) 444, 737 (2006); S. P. D. Mangles et al., Plasma Phys. Contr. Fus. 48, B83 (2006); J. Osterhoff et al., Phys. Rev. Lett. 101, 085002 (2008).

[5] N. H. Matlis et al., Nature Phys. 2, 749 (2006)

[6] A. G. R. Thomas et al., Phys. Rev. Lett. 98, 095004 (2007); J. E. Ralph et al., ibid. 102, 175003 (2009)

[7] J. Faure et al., Phys. Rev. Lett. 95, 205003 (2005)

[8] L. Gorbunov, P. Mora and T. M. Antonsen Jr, Phys. Rev. Lett. 76, 2495 (1996); Z. M. Sheng, J. Meyer-terVehn and A. Pukhov, Phys. Plasmas 5, 3765 (1998); P. Gibbon Short-Pulse Laser Interactions with Matter, (Imperial College Press, London, 2005).

[9] I. Kostyukov et al., Phys. Plasmas 11, 5256 (2004)

[10] W. Lu et al., Phys. Rev. Lett. 96, 165002 (2006) 
[11] K. Ta Phuoc et al., Phys. Rev. Lett. 97, 225002 (2006); A. G. R. Thomas et al., ibid. 98, 054802 (2007); S. Kneip et al., ibid. 100, 105006 (2008)

[12] B. Hidding et al., Phys. Rev. Lett. 96, 105004 (2006).

[13] M. C. Kaluza et al., Appl. Phys. B 92, 475 (2008)

[14] R. Benattar et al., Rev. Sci. Instr. 50, 1583 (1979).
[15] J. A. Stamper and B. H. Ripin, Phys. Rev. Lett. 34, 138 (1975).

[16] R. A. Fonseca et al. Lecture Notes in Computer Science Vol. 2329, III-342 (Springer, Heidelberg, 2002).

[17] F. S. Tsung et al., Phys. Rev. Lett. 93, 185002 (2004) 